\title{
Scutation asymmetries in red-footed tortoise Chelonoidis carbonaria Spix, 1824 (Testudines: Testudinidae)
}

\author{
Pere M. Parés-Casanova'; Pinzón Brando ${ }^{2,3}$; Daniel Caviedes ${ }^{2,4}$ \& Arcesio Salamanca-Carreño $0^{2,5}$ \\ ${ }^{1}$ University of Lleida, School of Agrifood and Forestry Science and Engineering, Department of Animal Science. Lleida, España. \\ ORCID: http://orcid.org/0000-0003-1440-6418. E-mail: peremiquelp@ca.udl.cat \\ ${ }^{2}$ Universidad Cooperativa de Colombia (UCC), Facultad de Medicina Veterinaria y Zootecnia. Arauca, Colômbia. \\ ${ }^{3}$ E-mail: donbranpinzon@gmail.com \\ ${ }^{4}$ ORCID: http://orcid.org/0000-0001-7871-7544.E-mail: daniel_3120@hotmail.es \\ ${ }^{5}$ ORCID: http://orcid.org/0000-0002-5416-5906. E-mail: asaca_65@yahoo.es
}

\begin{abstract}
The ability of an individual to withstand random perturbations during its development is considered a good indicator of environmental and genetic stress. A common means of assessing developmental stability is through analysis of fluctuating asymmetry (FA) in bilateral traits. Tortoises, with their large, solid plastron, allow for measurement of body geometry. Their bilateral shell scutes are ideal candidates for asymmetries researches. With this issue in mind we assessed, as a preliminary study, levels of plastron scute asymmetry in a sample of 46 red-footed tortoise Chelonoidis carbonaria from Arauca, N Colombia. We found significative fluctuating asymmetry (FA) but no directional asymmetry, the former not increasing with carapace size and thus indicating that tortoise shells do not become increasingly asymmetrical with age, or in other words, signaling that FA is not being influenced by pholidosis (variability of scale cover mosaic according to the development of the scutes). Asymmetry in plastron shape, although not necessarily apparent at first glance, varied, with gender with males exhibiting higher levels of FA than females. Although we can not identify the potential sources of variation responsible for the observed patterns of developmental instability, we consider this detected form of asymmetry due to unfavorable environmental conditions.
\end{abstract}

Key-Words. Directional asymmetry; Fluctuating asymmetry; Pholidosis; Tortoise shell.

\section{INTRODUCTION}

Bilaterally asymmetrical organisms can develop different kind of asymmetries, among which are fluctuating asymmetry (FA) and directional asymmetry (DA) (Graham et al., 1993). FA is defined as the deviation of the symmetry of an individual from perfect bilateral symmetry and is considered a negative indicator of the individual ability to resist random and small developmental accidents (Graham et al., 1993). These developmental accidents are generally the result of genetic or environmental stress (Auffray et al., 1999; Carter et al., 2009). On the other side, DA occurs whenever there is a greater development of a character on one side of the plane of symmetry than the other (Kharlamova et al., 2010; Lotto \& Béguelin, 2014). An important proportion of DA can have a genetic basis.

Tortoise shell represents the genotype by environment interaction of an individual throughout its life, being this genotype susceptible to environmental perturbations. In a typical case, scale cover mosaic of turtles (pholidosis) is arranged in longitudinal rows with strict bilateral symmetry (Cherepanov, 2014). But while the shell as an overall structure is under selection, specific landmarks on the shell (such as the location of scute sutures), may be under less direct selection (Băncilă et al., 2012; Buică \& Cogălniceanu, 2013; Cherepanov, 2014). To date, available studies of scale asymmetries are scarce and sometimes with contradictory conclusions. For instance, Dillard (Dillard, 2017) found no shell symmetric differences among two populations of Pseudemys species in east central Virginia, while Claude et al. considered them negligible, too (Claude et al., 2003). But Davis \& Grosse found FA in Trachemys scripta (Davis \& Grosse, 2008), as did Băncilă et al. (2012) in Testudo graeca ibera. Cherepanov (Cherepanov, 2014) describes a $81 \%$ of asymmetrical horny pattern in his studied collection, which included Mauremys caspica, Emys orbicularis, Testudo graeca and Agrionemys horsfieldi, while Buică \& Cogălniceanu (2013) detected low levels of FA in Testudo graeca. Moreover these latter authors ob- 
served no correlation of asymmetry with body size and suggested that FA would be an individual trait, with no predictive value at the population level. Finally, Rivera \& Claude (2008) suggested that turtle shell morphology may be less functionally constrained in terrestrial environments than in aquatic ones.

For the quantification of these asymmetries, both aspects of form (i.e., size and shape) should be assessed (Sforza et al., 1998). Size and shape mutually interact, but the variations in the former are often larger and easier to read than are the variations in the latter, which is more difficult to identify and quantify (Sforza et al., 1998). The two effects should, therefore, be separately quantified. Several approaches have been used for this purpose. In the present study we analyze them with geometric morphometric techniques, and in the plastron of red-footed tortoise (Chelonoidis carbonaria).

Chelonoidis carbonaria is a species from South America, distributed from northern Paraguay and Argentina through cis-Andean South America to Panama (Gallego-García et al., 2015; Cacciali et al., 2016; Turtle Taxonomy Working Group, 2017). These medium-sized tortoises generally average $30 \mathrm{~cm}$ as adults, but can reach over $40 \mathrm{~cm}$ (Barros et al., 2012). They have dark-colored, loaf-shaped carapaces (back shell) with a lighter patch in the middle of each scute, and dark limbs with brightly colored scales that range from pale yellow to dark red, although with a great variation in this morphological characteristic (Barros et al., 2012) (Gallego-García et al., 2015). No sex dimorphism is observed concerning size (Barros et al., 2012), but population differences are recognized between different regions (Barros et al., 2012). They are frequently kept as pets, and over-collection as well as habitat destruction have caused them to be vulnerable to extinction (Gallego-García et al., 2015).

Genetic or environmental disturbances that organisms can encounter during their embryonic development can be evaluated by FA. Because of the rapid and intense environmental change caused by anthropogenic activities in the Araucan "Ilanos" of Colombia, such as increasing conflicts of resource use and water quality deterioration, there is a growing local concern about the ecological consequences of human activities on animal populations. Given the need for this information, the present study aims to examine fluctuating asymmetry as indicator of fitness (Galeotti et al., 2005) based on the variation in plastral shape in Chelonoidis carbonaria from Arauca. We consider that plastron, due to its clear flatness, will present less coplanarity problems than carapace, although this fact has not been tested in this study.

\section{MATERIAL AND METHODS}

\section{Area sampling}

Arauca is a department of Colombia located in the extreme north of the Orinoco Basin of Colombia (the"Llanos Orientales"), bordering Venezuela. Its territory covers an area of 23,818 square kilometers. It is an ecosystem that

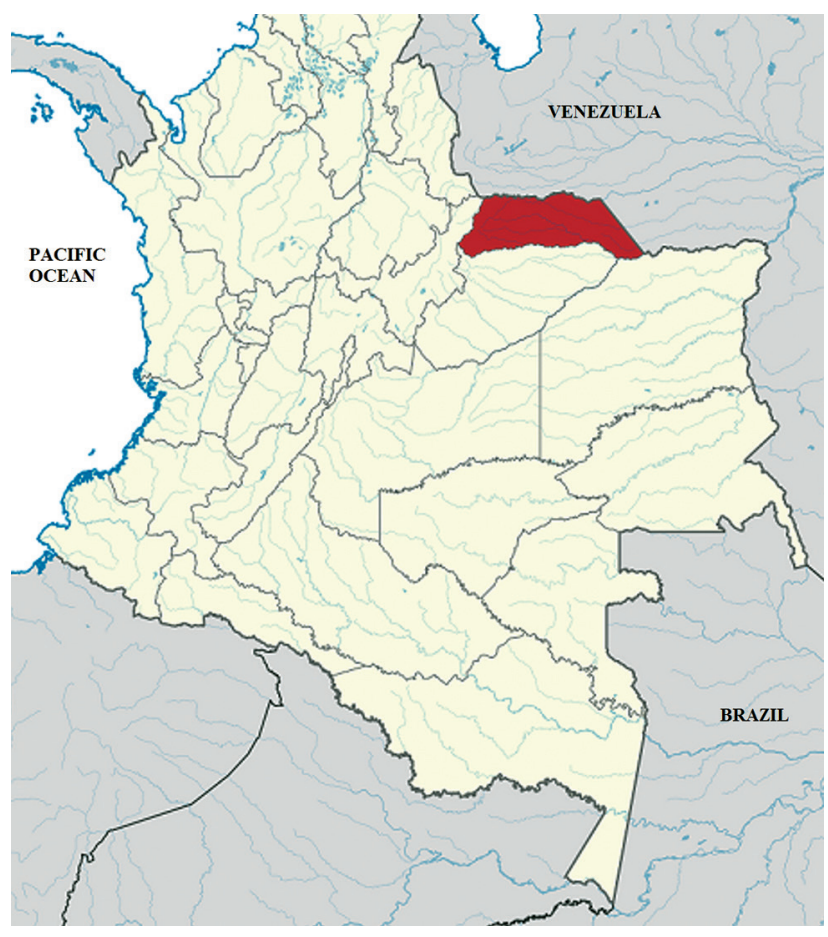

Figure 1. "Llanos Orientales", located between the rivers Arauca, Guaviare, Orinoco and Piedemonte Llanero, in Colombia.

is characterized by a plateau (Fig. 1). The region is located between the rivers Arauca, Guaviare, Orinoco and Piedemonte Llanero. Seventy-five per cent of the land is flat, but the eastern cordillera (mountain range) borders the western side of the territory where the Sierra Nevada of the Cocuy stands. At the foot of the cordillera, the soil is fertile and ideal for breeding livestock. The wet season is overcast, the dry season is muggy and mostly cloudy, and it is hot year round. Over the course of the year, the temperature typically varies from $72^{\circ} \mathrm{F}$ to $97^{\circ} \mathrm{F}$ and is rarely below $69^{\circ} \mathrm{F}$ or above $102^{\circ} \mathrm{F}$. Water is an important natural resource in Arauca, for there are many rivers such as the Arauca, Casanare, Tame and Tocoragua, among others. This enormous area with low touristic impact, although waste dumped by multinational companies are the main threats to the region.

\section{Data collection}

Data was obtained from 46 individuals of Chelonoidis carbonaria, during the dry season of February-March 2018. All the field-collected specimens displayed physical traits consistent with red-footed tortoise. All specimens used in the study were adults without any detectable abnormalities (such as injuries by predators, or unusual additional scutes or plates). Twelve of them were males (4.4-14.0 kg) and 34 were females (1.3-8.7 kg), with no differences in equality of distributions between males and females (according to Kolmogorov-Smirnov test $(D=0.392, p=0.09)$. We collected digital pictures in the field, holding the camera approximately $40-50 \mathrm{~cm}$ above each tortoise, with the camera focused on the central plastron aspect of the tortoise. It was obtained a single digital photograph of each animal. To prevent distortion 
and optical aberrations no zoom was used, and the tortoises were maintained without inclination, using props, to any side to reduce parallax errors. Identification of gender was based on the presence of a pronounced plastron concavity. Body weight was also obtained. Three individuals were not clearly sexed and they were excluded of the study. A standard was inserted on each image for image scalar measurement calibration. After their measurement and photographing, animals were then released at the site of capture. Second and third authors were responsible of this part. Images were posteriorly downloaded to a computer, and best landmarks for analyses selected. Shell length were 32.2 to $48.9 \mathrm{~cm}$ for males, 6.7 to $51.9 \mathrm{~cm}$ for females, so a wide range of age is supposed.

\section{Geometric morphometrics}

As plastrons are symmetric structures with object symmetry, characterized by an axis of symmetry that partially fit with the sagittal line, a landmark configuration without landmarks along the axial plane was tested (Fig. 2). The final symmetric shape was then obtained from eight landmarks (4 symmetric pairs) based on plastral sutures and scutes, landmarks, occurring on both sides of the plastron. These chosen landmarks were based on a previous studies on tortoises (Dillard, 2017) and were chosen in order to have a good representation of the overall shell scutes shape and in a way that allow us to see important features of asymmetry. Each picture was digitized at least twice, and an average of both configurations was taken. The captured images were transformed to TpsUtil software v. 1.40 (Rohlf, 2015a) and landmarks recorded using TpsDig v. 2.26 (Rohlf, 2010). We used TpsSmall v. 1.33 (Rohlf, 2015b) to test whether the observed variation in shape was sufficiently small that the distribution of points in the tangent space can be used as a good approximation of their distribution in shape space. Scale

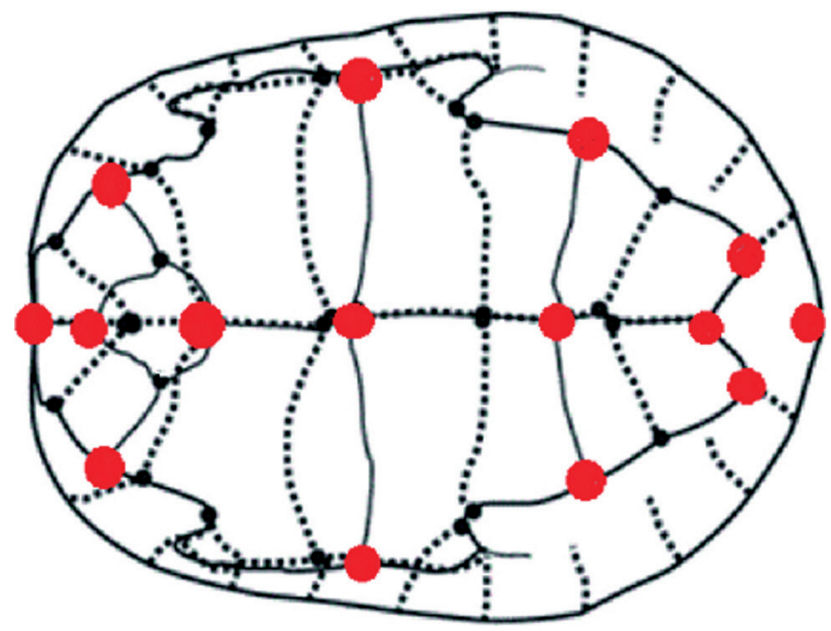

Figure 2. Landmarks situated (17) on sutures of plastral scutes (right: anal region). As plastron is a symmetric structure with object symmetry, characterized by an axis of symmetry that passes through the landmark configuration, the symmetry axis could partially fit with the sagittal line, the final landmark configuration excluded sagittal points, so remaining four paired landmarks. was eliminated by setting the centroid size, square root of sum of squared distances between the centroid and each landmark, the same in all specimens. Position was excluded by putting the centroid of every specimen at the same point. Orientation was taken away by rotating specimens so that square root of sum of squared distances between corresponding landmarks would be the minimum. By eliminating this information, only the shape was quantified for subsequent mathematical analysis.

Bilateral symmetry can either be displayed in the form of two separate mirrored copies (matching symmetry), or a single structure can be symmetric in itself and therefore possess an internal line or plane of symmetry (object symmetry). As a turtle shell has an internal plane of symmetry, we based our analyses on object symmetry. The set of original configurations and mirrored copies (including the replicated measurements) were then superimposed (scaled, translated, and rotated) following the Procrustes method of generalized least squares superimposition. Measurement error (ME) is of critical importance when analyzing FA (Palmer, 1994). To assess the significance of $F A$ relative to $M E$, all individuals were digitized twice on two different sessions.

\section{Statistical analyses}

To examine the amount of symmetric variation and asymmetry we used Procrustes ANOVA as usual in many studies on object symmetry (Klingenberg et al., 1998; Klingenberg \& Monteiro, 2005). To avoid the assumption of having an equal and independent variation at all points, it was performed a MANOVA test for symmetric component and asymmetry. This test is used to compare the individual reflection interactions to measurement error, the latter estimated from the total variation of the entire landmark configuration (Klingenberg, 2002). Then, the shape variation in the entire data set were assessed using principal component analysis (PCA), based on the covariance matrix of asymmetric components of shape variation. The first one is the average of left and right sides and represents the shape variation component, whereas the asymmetry component represents the individual left-right differences (Klingenberg, 2002). Finally, differences between sexes were assessed performing a canonical variate analysis (CVA), a multivariate statistical method used to find the shape characters that best distinguish among sexes, for both symmetric and asymmetric components. Analyses were done with MorphoJ v. 1.06c (Klingenberg, 2011). When needed, analyses were computed as permutation test with 10.000 permutation runs. For all analyses, $a=0.05$.

\section{RESULTS}

Analyses of the data set using TpsSmall indicated that an excellent correlation between the tangent and the shape space existed. The correlation (uncentered) between the tangent space, $Y$, regressed onto Procrustes distance was 0.9995 . There was little doubt on the ba- 
Table 1. ANOVA Procrustes analysis of plastron size and shape of red-footed tortoise Chelonoidis carbonaria with a significant interaction between the effects of individual and body side. The effect "side" can be interpreted as directional asymmetry and the interaction "individual *side" as fluctuating asymmetry. SS = Sum of Squares; MS = Mean Squares; $\mathrm{df}=$ degrees of freedom.

\begin{tabular}{lccccc}
\hline \multicolumn{1}{c}{ Effect } & SS & MS & df & F & p \\
\hline Individual & 0.2753655 & 0.0007171 & 384 & 8.10 & $<0.001$ \\
Side & 0.0005095 & $6.3698 \mathrm{E}-05$ & 8 & 0.72 & 0.674 \\
Individual * Side & 0.0339774 & $8.8483 \mathrm{E}-05$ & 384 & 1.43 & $<0.001$ \\
Error 1 & 0.0486097 & $6.2002 \mathrm{E}-05$ & 784 & & \\
\hline
\end{tabular}

sis of this result, which supported the hypothesis that samples can be analyzed by geometric morphometric methods since it was proved the acceptability of the data set for further statistical analysis. The estimate of shape was accurate as the measurement error was smaller than the true FA (MS values for individual $x$ side for shape: 0.000088483 in comparison with the individual value: 0.0007171 ) (Table 1). The repeat specimens clustered closely together on the principal axes relative to the variation between individuals, suggesting measurement error was small and the dispersal is isotropic in nature. The significant interaction of individual ${ }^{*}$ side (indicating individual variation in size and shape), confirmed the presence of significant presence of FA in the sample

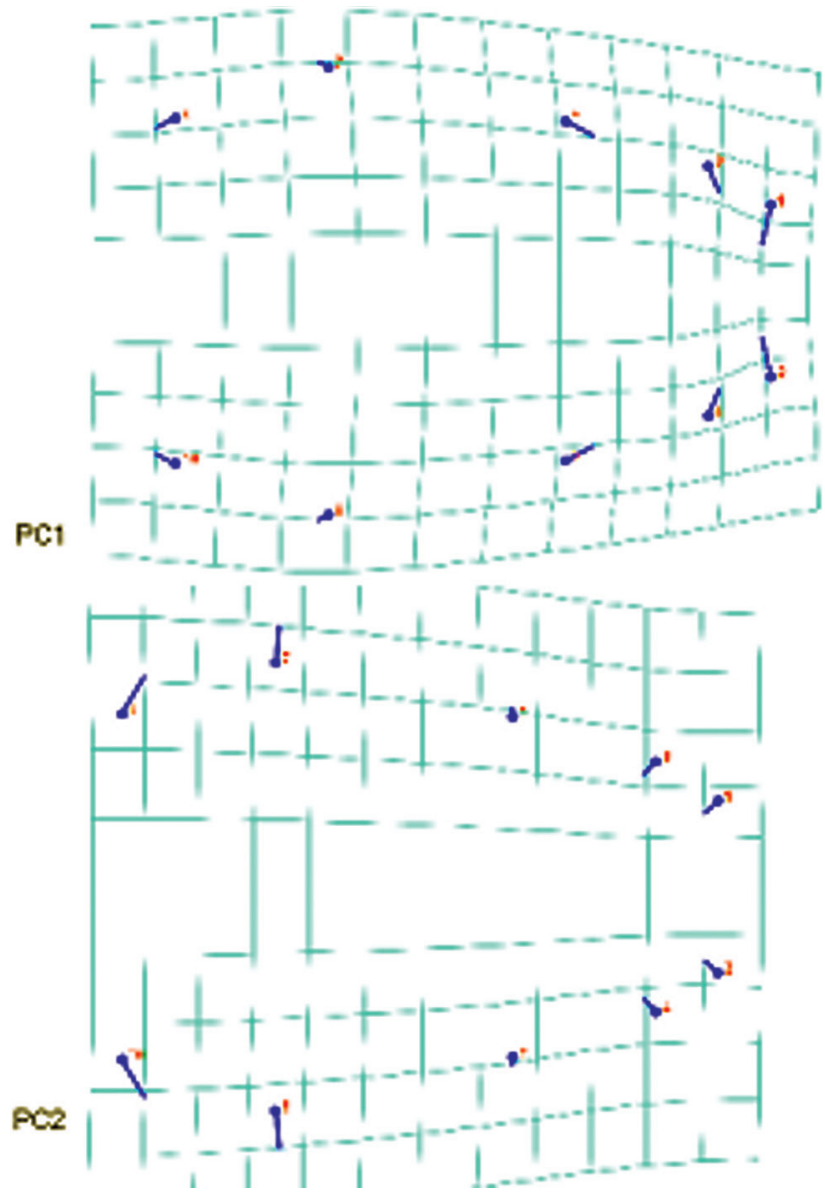

Figure 3. Symmetric components of shape variation. The first two PCs of the symmetric component were used to describe the pattern of symmetric part of shape variation, that is, the variation in the averages of the original and reflected landmark configurations constrained. The scores for PCs are arbitrary. $(p<0.001)$ but DA was not detected $(p=0.674)$. MANOVA test confirmed the presence of this FA being different between specimens (Pillai's trace $=6.58 ; p<0.001$ ). There was no change of symmetric components according to size change, shape variables and centroid size being found to be largely uncorrelated in the sample $(3.49 \%$; $p=0.165)$. CVA revealed sexual differences for symmetrical component $(p<0.0001)$, being levels higher in males.

PCA then was used with individually averaged values to identify and describe the pattern of individual asymmetries. The symmetric component of the variation (interpreted as FA), the first two PCs accounted for $65.67 \%$ of the total shape variation $(P C 1+P C 2=51.21 \%+14.46 \%)$ and the whole set of landmarks contribute quite equally to the variation among individuals (Fig. 3).

\section{DISCUSSION}

The use of geometric morphometrics plus multivariate statistical techniques provides a graphic visualization of the morphological variation of the individuals. In this study we used geometric morphometric approach to investigate the variation of body shape in a population of Chelonoidis carbonaria. Fluctuating asymmetry (FA) provides a useful metric of within-individual variance and is based on random departures from perfect symmetry. The two sides of a bilateral organism share the same genotype, yet each experiences small, random perturbations during the developmental process (Van Valen, 1962; Tomkins \& Kotiaho, 2001). These perturbations ultimately yield asymmetric phenotypes and since the direction of asymmetry is random in any given individual, it is described as fluctuating (Palmer, 1996; Mancini et al., 2005). Unlike most ecological, physiological or developmental responses, FA provides an ideal state (i.e., perfect symmetry), making it a suitable measure for evaluating environmental stress. However, any real-world population is expected to show some level of variance due to developmental noise, therefore the null hypothesis is that the shape (i.e., variance) of the right-left sides of a trait's distribution is equal for tortoises. But our analyses showed significative degrees of plastron FA, with a morphological differentiation among sexes.

Does this significant FA suggest a certain degree of stress levels caused by unfavorable environmental factors, which may directly influence plastron shape? The missing correlation for body size and FA leaves the observed variations in asymmetry open to speculation. The breaking of turtle pholidosis symmetry is connected with deviations in ontogenesis, as signaled by Cherepanov (2014). Aberrant symmetries are apparently not determined genetically nor influence individual viability (Cherepanov, 2014). It is frequently suggested that morphological variation of individuals may be strongly dependent upon unfavorable environmental conditions, and in our sample a non-age-related action of potential stressors, which could interfere with normal symmetrical shell production along growth (e.g., size increase) of animals is detected. In other words, such FA would emerge not from plasticity or adaptation, but from the affected to development 
for this species, at least in the tortoises from the Arauca area. The differences in the scutes from the left and right sides are consistent with the data obtained by many other researches performed in turtles and tortoises (Davis \& Grosse, 2008; Macchi et al., 2008; Buică \& Cogălniceanu, 2013; Cherepanov, 2014; Claude, 2016).

Self-righting, the capacity of an animal to self-turn after falling on its back, which is a fitness-related trait (Domokos \& Varkonyi, 2008; Chiari et al., 2017), is rejected, as no correlation was observed between extracted size and residual shape variables, as it would be logical if the FA had a this functional sense. The possibility of being a secondary sexual characters is also rejected, although being different between males and females, as it would need to appear with a negative relationship (larger sexual traits are presumably more costly to produce, which should lead to greater developmental stress and corresponding increases in asymmetry) (Jennions, 1996). This sexual dimorphism in FA has been signaled by other authors, e.g., as did Băncilă et al. (2012) in Testudo graeca ibera. In the absence of negative relationship, it is also suggested that FA expression is non condition-dependent (Jennions, 1996). A potential consequence of higher $F A$, as indicator of the loss of developmental stability, is that it can allow to express cryptic genetic diversity into divergent phenotypes.

The stability of the scute pattern is connected with the basic mechanisms of morphogenesis, providing the maintenance of bilateral symmetry and determination of scute positions (Cherepanov, 2014). The main source for pholidosis variability is due to placodes which could have developed in the case of minor disturbances of embryogenesis, which would result in asymmetry of the scutation (Cherepanov, 2014). DA did not appear in the studied sample. But the evolution of anthropogenic activities and changes in environmental factors in the studied area are dynamics must be considered in future studies of developmental instabilities. Ideally a larger sample size and a wider age range would support stronger conclusions, and this will be considered for further studies, the present being considered just as a preliminary one.

\section{AUTHORS' CONTRIBUTIONS}

PMPC and ASC designed the experiments, analyzed the data and wrote the paper. PB and DC performed the field study.

\section{COMPETING INTERESTS}

We have no competing interests.

\section{ACKNOWLEDGEMENTS}

The authors wish to declare our most sincere acknowledgement to Editor and reviewers of the Journal, as well as to Araucan owners who allowed the access to their proprieties.

\section{REFERENCES}

Auffray, J.C.; Debat, V. \& Alibert, P. 1999. Shape asymmetry and developmental stability. In: Mark, A.J.; Chaplain, G.D. \& Singh, J.C.M. (Ed.). On growth and form: spatio-temporal pattern formation in biology. New York, John Wiley and Sons Ltd. p. 309-324.

Băncilă, R.I.; Plăiaşu, R.; Tudor, M.; Samoilă, C. \& Cogălniceanu, D. 2012. Fluctuating Asymmetry in the Eurasian Spur-Thighed Tortoise, Testudo graeca ibera Linneaus, 1758 (Testudines: Testudinidae). Chelonian Conservation and Biology, 11(2): 234-239.

Barros, M.S.; Resende, L.C.; Silva, A.G. \& Ferreira Junior, P.D. 2012. Morphological variations and sexual dimorphism in Chelonoidis carbonaria (Spix, 1824) and Chelonoidis denticulata (Linnaeus, 1766) (Testudinidae). Brazilian Journal of Biology, 72(1): 153-161. D0I

Buică, G. \& Cogălniceanu, D. 2013. Using digital images in the study of fluctuating asymmetry in the spur-thighed tortoise Testudo graeca. Turkish Journal of Zoology, 37(6): 723-729. DOI

Cacciali, P.; Scott, N.J.; Ortíz, A.L.A.; Fitzgerald, L.A. \& Smith, P. 2016. The Reptiles of Paraguay: Literature, Distribution, and an Annotated Taxonomic Checklist. Special Publication of the Museum of Southwestern Biology, 11: 1-373.

Carter, A.J.R.; Osborne, E. \& Houle, D. 2009. Heritability of directional asymmetry in Drosophila melanogaster. International Journal of Evolutionary Biology, 1-7. D0I

Cherepanov, G.0. 2014. Patterns of scute development in turtle shell: Symmetry and asymmetry. Paleontological Journal, 48(12): 1275-1283. D0l

Chiari, Y.; van der Meijden, A.; Caccone, A.; Claude, J. \& Gilles, B. 2017. Self-righting potential and the evolution of shell shape in Galápagos tortoises. Scientific Reports, 7(1): 1-8. DOI

Claude, J. 2016. Environmental media and shape asymmetry: a case study on turtle shells. Biological Journal of the Linnean Society, 94(3): 483-489 + 1 pl. DOI

Claude, J.; Paradis, E.; Tong, H. \& Auffray, J.-C. 2003. A geometric morphometric assessment of the effects of environment and cladogenesis on the evolution of the turtle shell. Biological Journal of the Linnean Society, 79(3): 485-501. D0I

Davis, A.K. \& Grosse, A.M. 2008. Measuring Fluctuating Asymmetry in Plastron Scutes of Yellow-Bellied Sliders: The Importance of Gender, Size and Body Location. The American Midland Naturalist, 159(2): 340-348. D0I

Dillard, K.C. 2017. A comparative analysis of geometric morphometrics across two Pseudemys turtle species in east central Virginia. Richmond, Virginia Commonwealth University. 81p. (Thesis of Master Degree)

Domokos, G. \& Varkonyi, P.L. 2008. Geometry and self-righting of turtles. Proceedings of the Royal Society B: Biological Sciences, 275(1630): 11-17. D01

Galeotti, P.; Sacchi, R. \& Vicario, V. 2005. Fluctuating asymmetry in body traits increases predation risks: tawny owl selection against asymmetric woodmice. Evolutionary Ecology, 19(4): 405-418. DOI

Gallego-García, N.; Cárdenas-Arévalo, G. \& Castaño-Mora, 0.V. 2015. Chelonoidis carbonaria (Spix 1824). In: Morales-Betancourt, M.A.; Lasso, C.A.; Páez, V.P. \& Bock, B.C. (Ed.). Libro Rojo de Reptiles en Colombia. Bogotá, Colombia, Instituto de Investigación de Recursos Biológicos Alexander von Humboldt. p. 406-411.

Graham, J.H.; Freeman, D.C. \& Emlen, J.M. 1993. Antisymmetry, directional asymmetry, and dynamic morphogenesis. Genetica, 89(1-3): 121-137. DOI

Jennions, M. 1996. The allometry of fluctuating asymmetry in southern African plants: flowers and leaves. Biological Journal of the Linnean Society, 59(2): 127-142. 
Kharlamova, A.V.; Trut, L.N.; Chase, K.; Kukekova, A.V. \& Lark, K.G. 2010. Directional asymmetry in the limbs, skull and pelvis of the silver fox (V. vulpes). Journal of Morphology, 271(12): 1501-1508.

Klingenberg, C.P. 2002. Morphometrics and the role of the phenotype in studies of the evolution of developmental mechanisms. Gene, 287(1-2): 3-10.

Klingenberg, C.P. 2011. MorphoJ: An integrated software package for geometric morphometrics. Molecular Ecology Resources, 11(2): 353-357.

Klingenberg, C.P. \& Monteiro, L.R. 2005. Distances and directions in multidimensional shape spaces: implications for morphometric applications. Systematic Biology, 54(4): 678-688. DOI

Klingenberg, C.P.; McIntyre, G.S. \& Zaklan, S.D. 1998. Left-right asymmetry of fly wings and the evolution of body axes. Proceedings of the Royal Society B: Biological Sciences, 265(1402): 1255-1259. D0I

Lotto, F. \& Béguelin, M. 2014. Asimetría direccional del postcráneo en poblaciones prehispánicas del sur de Sudamérica. Antropología Biológica, 7(1): 133-142.

Macchi, S.; Balzarini, L.; Scali, S.; Martinoli, A. \& Tosi, G. 2008. Assessing shell asymmetry in Trachemys scripta using geometric morphometrics. In: Congresso Nazionale Societas Herpetologica Italica, $7^{\circ}$. Herpetologie sardiniae. Oristano, Edizione Belvedere. p. 333-337.

Mancini, S.; Sally, S.L. \& Gurnsey, R. 2005. Detection of symmetry and antisymmetry. Vision Research, 45(16): 2145-2160.

Palmer, A.R. 1994. Fluctuating asymetry analysis: a primer. In: Markow, T.A. (Ed.). Developmental instability: its origins and evolutionary implications. Netherlands, Kluwer. p. 335-364.
Palmer, A.R. 1996. From symmetry to asymmetry: phylogenetic patterns of asymmetry variation in animals and their evolutionary significance. Proceedings of the National Academy of Sciences of the United States of America, 93(25): 14279-14286.

Rivera, G. \& Claude, J. 2008 Environmental media and shape asymmetry: a case study on turtle shells. Biological Journal of the Linnean Society, 94: 483-489.

Rohlf, F.J. 2010. Digitalized Landmarks and Outlines. 2.26. New York, Stony Brook: Department of Ecology and Evolution, State University of New York.

Rohlf, F.J. 2015a. The tps series of software. Hystrix, the Italian Journal of Mammalogy, 26(1): 9-12.

Rohlf, F.J. 2015b. TpsSmall v. 1.33. Available at: http://life.bio.sunysb.edu/ morph.

Sforza, C.; Michielon, G.; Fragnito, N. \& Ferrario, V.F. 1998. Foot asymmetry in healthy adults: Elliptic fourier analysis of standardized footprints. Journal of Orthopaedic Research, 16(6): 758-765.

Tomkins, J.L. \& Kotiaho, J.S. 2001. Fluctuating asymmetry. In: Encyclopedia of Life Sciences. London, MacMillan. p. 1-5.

Turtle Taxonomy Working Group (TTWG). 2017. Turtles of the world. Annotated checklist and atlas of taxonomy, synonymy, distribution and conservation status. 8. ed. Lunenburg, Chelonian Research Foundation and Turtle Conservancy. 296p. (Chelonian Research Monographs, 7). Available at: http://images.turtleconservancy.org/documents/2017/crm-7-checklistatlas-v8-2017.pdf.

Van Valen, L. 1962. A study of fluctuating asymmetry. Evolution, 16: 125-142. 